\title{
Gastrectomía atípica laparoscópica
}

\section{Laparoscopic atypical gastrectomy}

Camila Haro ${ }^{1}$

ORCID:https://orcid.org/0000-0003-4475-9740

Nicolás Muniz ${ }^{2}$

ORCID: https://orcid.org/0000-0002-6235-1303

Nicolás González ${ }^{3}$

ORCID: https://orcid.org/0000-0002-7785-3963

Andrés Pouy ${ }^{4}$

ORCID: https://orcid.org/0000-0002-7136-144X

Santiago Cubas ${ }^{5}$

ORCID: https://orcid.org/0000-0001-6026-5316

DOI: $10.31837 /$ cir.urug/5.2.2

Recibido: 12 de junio de 2020

Aceptado: 08 de febrero de 2021

\section{Introducción}

El cáncer gástrico es la quinta neoplasia maligna más frecuente a nivel mundial y en nuestro país, y la cuarta causa de mortalidad por cáncer.

La tasa de supervivencia continúa siendo baja, dado que su diagnóstico suele ser tardío y en etapas avanzadas.

\section{GASTRECTOMÍA ATÍPICA LPSC}

Dres. Haro C, Muniz N, Gonzalez N, Pouy A, Cubas S. Clinica Quinürgica "B"- Clínica de Gastroenterologla Hospital de Clinicas

En países con alta incidencia se realiza screening endoscópico, realizándose el diagnóstico en etapas precoces, con un mejor pronóstico. En estos pacientes existe menor riesgo de diseminación regional o a

\footnotetext{
1,2,4,5 Universidad de la República, Facultad de Medicina, Hospital de Clínicas, Clínica Quirúrgica B. harob.camila@gmail.com

${ }^{3}$ Universidad de la República, Facultad de Medicina, Hospital de Clínicas, Clínica de Gastroenterología
} 
distancia, y se pueden plantear tratamientos a medida, evitando resecciones amplias.

El cáncer gástrico precoz se define como aquel en el que la invasión tumoral está confinada a la mucosa o submucosa (T1), independientemente del compromiso glanglionar. Datos japoneses muestran una sobrevida global del 99\% a 5 años para aquellos confinados a la mucosa y 96\% en los que invaden la submucosa. La profundidad de la invasión determinará también el riesgo de compromiso ganglionar, que es del 3\% en la enfermedad limitada a la mucosa, y llega al 20\% cuando ésta se extiende a la submucosa.

Se han desarrollado múltiples técnicas miniinvasivas para el tratamiento de los tumores precoces, incluyendo la resección mucosa o submucosa por vía endoscópica, abordajes laparoscópicos o robóticos.

Como las resecciones endoscópicas no se acompañan de linfadenectomía, es importante la evaluación de su indicación, reservándose para tumores intramucosos pequeños, de tipo intestinal, en los que el compromiso ganglionar es poco probable.

Las lesiones de gran tamaño de tipo histológico difuso tienen más riesgo de invasión submucosa y metástasis ganglionares, lo que los hace malos candidatos para este tipo de resección.

Las complicaciones de la resección endoscópica incluyen: dolor, sangrado y perforación. Las perforaciones suelen poder cerrarse con clips, sin requerir nuevas intervenciones. El lecho submucoso luego de la resección cierra en aproximadamente 6 a 8 semanas, y los pacientes deben seguir un tratamiento con IBP por ese período de tiempo.

La recurrencia luego de la resección endoscópica es de aproximadamente 6\%.

Aunque la gastrectomía con linfadenectomía sigue siendo el tratamiento gold standard para la mayor parte de los cánceres gástricos, el advenimiento de la LPSC ha permitido el desarrollo de nuevas técnicas como: gastrectomía laparoscópica (LPSC), resección mucosa laparoscópica intragástrica y resecciones atípicas.

Los procedimientos resectivos han sido modificados con el objetivo de disminuir las secuelas funcionales de las resecciones gástricas radicales, como el dumping, gastroesofagitis por reflujo y pérdida de peso.

La resección mucosa laparoscópica intragástrica fue inicialmente descripta por Ohashi et al, y requiere la colocación de 3 trócares con balón en la cavidad abdominal y, a través de la misma, en la luz gástrica. A través de uno de los trocares se introduce la cámara, y en los dos restantes el instrumental. Se requieren trócares con balón para prevenir la salida de aire a través de la pared gástrica. Esta técnica puede utilizarse para tumores en cualquier topografía salvo en la pared anterior del estómago.

La resección puede realizarse mediante el método de “levantamiento de la lesión”, seccionándola mediante una grapadora, o mediante disección seromuscular. 
La gastrectomía LPSC es cada vez más utilizada para el cáncer gástrico con potencial compromiso ganglionar, con beneficios sobre la cirugía abierta que incluyen una menor pérdida sanguínea, menor morbilidad parietal, menor repercusión respiratoria, y más rápida recuperación postoperatoria. Existen estudios que demuestran que la misma presenta resultados oncológicos comparables con la cirugía abierta. Debemos tener en cuenta que debe ser realizada por un equipo entrenado y siguiendo los mismos principios oncológicos. Se ha demostrado que la linfadenectomía LPSC conlleva un mayor tiempo operatorio y requiere de mayores habilidades técnicas que la realizada por vía abierta, con una curva de aprendizaje que llega a las 50 cirugías.

La combinación de técnicas laparoscópicas y endoscópicas fue desarrollada por Hiki et al, para la disección de tumores gástricos submucosos. Esta técnica implica identificación endoscópica de tumor y topografía del mismo, seguido de disección endoscópica submucosa. Se realiza una disección serosa endoscópica y se cierra la línea de incisión con una grapadora mecánica. Esta técnica se utiliza en tumores mayores a 3cm, localizados en la curvatura mayor o en lesiones con ulceraciones extensas. Destacamos que esta técnica no se acompaña de linfadenectomía.

Se ha investigado la combinación de técnicas de resección endoscópica con linfadenectomía LPSC en casos en los que no se puede descartar el compromiso ganglionar. Se han visto complicaciones asociadas al mismo como isquemia por sección arterial durante la linfadenectomía o retardo en el vaciamiento gástrico, por lo que por el momento no es un procedimiento estándar.

La biopsia de ganglio centinela se utiliza con frecuencia en tumores como melanoma y cáncer de mama. En los últimos años se ha propuesto su uso en cáncer gástrico, en vistas a evitar linfadenectomías extensas en tumores precoces, candidatos a resecciones limitadas. Dado que el drenaje linfático gástrico puede seguir varias vías, y existe un número significativo de metástasis salteadas (skip metástasis), este estudio tiene limitaciones y no se ha extendido su uso.

\section{Caso clínico}

Hombre de 67 años.

AP: HTA en tratamiento con Enalapril. Cardiopatía isquémica, CACG en 2011 con colocación de 2 stents. DM tipo 2 insulinorequiriente. ERC estadio IIIb. Ex tabaquista, no BC.

AQ: Colecistectomizado en 2003 por vía abierta. Colectomía derecha en 2017 por adenocarcinoma moderadamente diferenciadeo de ciego. Nefrectomía izquierda LPSC en 2018 por cáncer de células claras.

En noviembre 2019, durante FGC indicada por epigastralgia se evidencia a nivel desinusangularis, sobre vertiente vertical de curvatura menor lesión elevada y deprimida de $20 \mathrm{~mm}$, de bordes irregulares, con retracción de la mucosa y convergencia de pliegues a dicho nivel. Se biopsia, revelando el estudio anátomopatológico un adenocarcinoma gástrico intramucoso bien diferenciado, con foco sospechoso pero no concluyente de invasión de la muscularismucosae. 
A nivel de antro sobre cara posterior otra lesión de 10mm, deprimida, con bordes ligeramente elevados, que se biopsia. La anatomía patológica de la misma revela un adenoma gástrico con displasia de bajo grado y de alto grado focal.

Resto de la mucosa con aspecto de atrofia y metaplasia intestinal.El estudio anátomo-patológico revela una gastritis crónica atrófica.

Se realiza una nueva endoscopía con tinción con índigo carmín y cromo-endoscopía digital y magnificación. Se evalúan las lesiones, sin observarse sectores sugestivos de invasión submucosa profunda.

Con estos hallazgos se decide resección endoscópica submucosa. Se realiza una nueva endoscopía con tinción índigo carmín, que permite el marcado de la mucosa sana mediante electrocauterio. Posteriormente se eleva la mucosa mediante inyección submucosa de SF. Se comienza la sección mucosa y submucosa, pero al llegar al sector central de la lesión se observa "muscleretreatingsign” y fibrosis severa, sugestivos de invasión submucosa profunda, se decide no proseguir con el procedimiento y se deriva a cirujano para resolución quirúrgica.

Una vez valorado por equipo de cirugía se solicita TC de tórax, abdomen y pelvis para estadificación oncológica. Se descartan recidivas de neoplasias previas, lesiones secundarias hepáticas o pulmonares y adenopatías sospechosas de malignidad, estadificándose como un cT1bN0M0.

Se realiza procedimiento combinado laparoscópico - endoscópico. Mediante endoscopía se topografía la lesión, permitiendo el marcado LPSC de la misma con márgenes oncológicos.

Posteriormente se incide la pared gástrica, resecando un medallón parietal que se extrae por medio del endoscopio, y posteriormente realizando una resección submucosa de la lesión de menor tamaño en la cara posterior gástrica.

Para finalizar se realiza el cierre borde a borde del defecto gástrico con V-lock, mediante sutura LPSC.

El paciente presenta una buena evolución postoperatoria, otorgándose el alta a la semana del procedimiento.

La anatomía patológica de la pieza de resección informa un adenocarcinoma bien diferenciado de tipo intestinal de Lauren, de 28x20mm, que asienta en un adenoma de 35x30mm. El mismo es predominantemente intramucoso con focos de invasión de la submucosa de hasta $1 \mathrm{~cm}$ de profundidad. No presenta embolias vasculares (pT1bNxMx). El margen de resección más cercano está a 3mm.

La segunda lesión se trata también de un adenocarcinoma bien diferenciado intramucoso, con invasión submucosa focal menor a 500 micras (pT1bNxMx). El margen de resección lateral pasa por tumor, el margen profundo es de $0,6 \mathrm{~mm}$. 